\title{
Protective Effect of Tegaserod Against Indomethacin-Induced Gastric Injury in the Rat
}

\author{
Kalina Venkova ${ }^{\mathrm{b}}$, David L. Earnest ${ }^{\mathrm{c}}$ and Beverley Greenwood-Van Meerveld ${ }^{*, \mathrm{a}, \mathrm{b}}$ \\ ${ }^{a}$ VA Medical Center, ${ }^{b}$ Oklahoma Center for Neuroscience, University of Oklahoma Health Science Center, Oklahoma \\ City, OK 73104, USA \\ ${ }^{c}$ Novartis Pharmaceuticals Corporation, One Health Plaza, East Hanover, NJ 07936, USA
}

\begin{abstract}
Gastric contractions induced by non-steroidal anti-inflammatory drugs (NSAIDs) contribute to mucosal ulceration. The goal of the present study was to investigate whether maintaining normal gastric motility provides protection against NSAIDs. A rat model of indomethacin-induced gastric mucosal injury and muscle hypercontratility was used to evaluate the protective effect of pretreatment with the $5-\mathrm{HT}_{4}$ receptor agonist tegaserod $(1 \mathrm{mg} / \mathrm{kg}$ b.i.d.) in comparison to the effect of the proton pump inhibitor omeprazole $(20 \mathrm{mg} / \mathrm{kg}$ b.i.d). Indomethacin induced mucosal ulceration associated with hypercontractility of isolated antral muscle strips in response to $\mathrm{KCl}$, carbachol, 5-HT or electrical field stimulation. Pretreatment with tegaserod alone or in combination with omeprazole prevented both mucosal ulceration and muscle hypercontractility. In contrast, omeprazole protected the mucosa without preventing the development of muscle hypercontractility. The results show that activation of peripheral 5- $\mathrm{HT}_{4}$ receptors promotes normal contractility of the antrum suggesting a different mechanism of gasrtoprotection against NSAIDs.
\end{abstract}

\section{INTRODUCTION}

Nonsteroidal anti-inflammatory drugs (NSAIDs), which act as non-selective cyclooxygenase (COX) inhibitors, are commonly used to treat pain and inflammation despite the risk of major upper gastrointestinal complications. The primary cause of the pathogenic effects of NSAIDs in the stomach is the deficiency of endogenous prostaglandins required to maintain the mucosal barrier to luminal acid [1-4]. Oxygen free radicals and lipid peroxidation [5], leukocyte adhesion and tumor necrosis factor alpha [6] were found to play an important role in this process. However, a breakdown in the mucosal barrier against gastric acid is not the only mechanism contributing to mucosal injury during NSAIDs administration. Studies using rat models of NSAID treatment have shown alterations in gastroduodenal motility which result in delayed gastric emptying and mucosal ulceration [7, 8]. Oral administration of indomethacin in rats $[9,10]$ and dogs [11] was found to induce gastric muscle hypercontractility which interferes with the motor function of the stomach and contributes to the development of mucosal injury.

To avoid NSAID-induced gastrointestinal complications, patients with one or more risk factors (history of ulcer disease, high doses or use of multiple NSAIDs, advanced age etc.) receive concurrent gastroprotective therapy. The maximal protective effects are found clinically with proton pump inhibitors prescribed as co-therapy with the NSAID. Omeprazole $(20 \mathrm{mg} /$ day $)$ has been shown to prevent recurrence of ulcer bleeding in high-risk patients $[12,13]$. The mechanism responsible for the protective effect of proton pump inhibitors is the suppression of gastric acid release, which leads to a less injurious environment at the gastroduodenal mucosal

*Address correspondence to this author at the Oklahoma Center for Neuro-
science, University of Oklahoma Health Sciences Center, 921 NE 13th
Street, Oklahoma City, OK 73104, USA; Tel: 405-270-0501, Ext. 3547; Street, Oklahoma City, OK 73104, USA; Tel: 405-270-050 surface. However, proton pump inhibitors have no protective effect against the NSAID-induced gastric muscle contractility, which contributes to the development of mucosal ulcers [14]. Moreover, omeprazole itself has been shown to cause a delay in gastric emptying in healthy subjects [15-19]. Thus, the various mechanisms involved in NSAIDs-induced gastric injury raise an important question whether the pharmacological targeting of receptors which induce peristalsis and promote "normal" gastrointestinal motility may have a protective effect. 5-Hydroxytryptamine-4 $\left(5-\mathrm{HT}_{4}\right)$ receptors are activated by drugs like tegaserod and renzapride, which have been used to treat symptoms in constipated irritable bowel syndrome patients and in patients with gastroparesis [20]. Although tegaserod has been withdrawn from the market because of cardiovascular side effects, new serotonin modulators targeting $5-\mathrm{HT}_{4}$ or $5-\mathrm{TH}_{3}$ receptors are still in the main focus of drug development because of their ability to initiate peristaltic and secretory reflexes in the gastrointestinal tract and their role in conveying sensory information to the central nervous system [21]. Thus, the goal of our study was to investigate whether tegaserod, a prokinetic acting as a potent partial agonist of peripheral $5-\mathrm{HT}_{4}$ receptors [22] and modest antagonist of $5-\mathrm{HT}_{1 \mathrm{~B}}$ and $5-\mathrm{HT}_{2 \mathrm{~B}}$ receptors $[23,24]$ has a protective effect against NSAID-induced gastric muscle dysfunction and mucosal injury. The experiments employed a rat model of indomethacin-induced gastric mucosal ulceration and smooth muscle dysfunction. To gain a better perspective of the possible gastroprotective action of prokinetics and their application with other therapies, the effects of the proton pump inhibitor omeprazole and a combination of tegaserod with the proton pump inhibitor were also investigated.

\section{METHODS}

Animals. Male Sprague-Dawley rats (Charles River, Laboratories, Wilmington, MA) weighing $250-300 \mathrm{~g}$ were housed two in a cage at standard conditions $\left(21-23^{\circ} \mathrm{C}\right.$, con- 
trolled humidity, $12 \mathrm{hr}$ light/dark cycle). The animals were allowed to acclimate for one week prior to initiation of drug treatment. The experimental procedures were approved by the Animal Care and Use Committees at the V.A. Medical Center and the University of Oklahoma Health Science Center, Oklahoma City, OK.

Indomethacin-induced gastric damage. Prior to the experiment the rats were fasted overnight with free access to water. On the day of the experiment, the rats received an intragastric gavage of $50 \mathrm{mg} / \mathrm{kg}$ indomethacin dissolved in $2 \% \mathrm{NaCO}_{3}$ and brought to a volume of $1.5 \mathrm{ml}$ with saline (pH 6.8). Indomethacin was administered at 7:30 AM and the animals were placed in their home cages with free access to food and water. Six hours after indomethacin dosing, the rats were euthanized, the stomach was isolated and placed in $\mathrm{Ca}^{2+}$-free Krebs buffer aerated with $95 \% \mathrm{O}_{2}$ and $5 \% \mathrm{CO}_{2}$.

Drug treatment. Tegaserod and omeprazole were dissolved in propylene glycol. Drugs were prepared fresh for each experiment and stored at $4^{\circ} \mathrm{C}$ between treatments. Prior to administration of indomethacin the rats were randomly assigned to groups (6 rats per group) receiving the following treatments: 1) $1 \mathrm{mg} / \mathrm{kg}$ tegaserod, 2) vehicle (propylene glycol), 3) $20 \mathrm{mg} / \mathrm{kg}$ omeprazole or 4) a combination of $1 \mathrm{mg} / \mathrm{kg}$ tegaserod and $20 \mathrm{mg} / \mathrm{kg}$ omeprazole. The drugs or vehicle alone were administered intraperitoneally in a volume of 0.1 $\mathrm{ml} / 100 \mathrm{~g}$ body weight at both $8 \mathrm{AM}$ and $5 \mathrm{PM}$ one day prior to the experiment and again on the day of experiment $30 \mathrm{~min}$ prior to the intragastric dosing with indomethacin. A group of naïve untreated rats $(n=6)$ was used as a reference to healthy controls.

Evaluation of mucosal injury. The stomach was cut along the lesser curvature, cleansed of ingested food and the fundus separated from the glandular portion of the stomach. Both regions were pinned flat to the silgard-covered bottom of a dish filled with $\mathrm{Ca}^{2+}$-free Krebs buffer which was continuously aerated with $95 \% \mathrm{O}_{2}$ and $5 \% \mathrm{CO}_{2}$ and then placed on the stage of a dissecting microscope. Mucosal ulceration was observed as redness and bleeding (areas about $1 \mathrm{~mm}$ wide and of different length) along the surface of mucosal folds. Ulceration was pronounced in the glandular portion of the stomach but absent in the fundic area. The length of each hemorrhagic lesion was measured and the total length $(\mathrm{mm})$ served as an index of ulceration. Subsequently, the mucosa was carefully dissected from the muscle, snap-frozen in liquid nitrogen and stored at $-80^{\circ} \mathrm{C}$. The investigator evaluating mucosal injury was blinded with regard to the treatment.

Myeloperoxidase activity. Myeloperoxidase (MPO) was measured in gastric mucosa as a marker of neutrophil infiltration reflecting inflammation. Mucosal samples were thawed on ice for analysis of MPO activity. Homogenization and extraction of MPO from the homogenate by subsequent freeze-thaw sessions were carried out in hexadodecyltrimethylamonium bromide (HTAB) phosphate buffer $(\mathrm{pH}$ 6). MPO activity was tested in $10 \mu 1$ samples using 3,3',5,5'tetramethylbenzidine TMB Microwell Peroxidase Substrate System (Sigma, St. Louis, MO) and horseradish peroxidase (HRP) as a relative standard. MPO activity was expressed as equivalent to the activity of the relative standard (ng of HRP) converting the same amount of TMB substrate for $10 \mathrm{~min}$ at room temperature. The data were expressed in ng normalized per $\mathrm{g}$ wet weight of the tissue.
Smooth muscle contractility. Krebs buffer free of $\mathrm{Ca}^{2+}$ was used during tissue isolation to minimize muscle contractility and avoid overstretching. Circular smooth muscle strips $(10 \mathrm{~mm} \times 2 \mathrm{~mm})$ were isolated from the gastric antrum and suspended in organ baths under an initial load of $0.5 \mathrm{~g}$ (5 $\mathrm{mN}$ ). The bathing solution was then switched to Krebs containing $2.5 \mathrm{mM} \mathrm{Ca}{ }^{2+}$ and isometric tone and spontaneous phasic contractions were recorded after a 90 -min equilibration. Optimal tension was adjusted by loading the preparation and examining the contractile response to $80 \mathrm{mM} \mathrm{KCl}$. The experimental protocols were designed to characterize indomethacin-induced changes in smooth muscle contractility and the ability of tegaserod, omeprazole and the combination to prevent smooth muscle dysfunction. Experiments were performed to investigate: contractile responses induced by receptor-independent $\mathrm{KCl}$ depolarization; concentrationresponse curves to carbachol or 5-HT due to activation of smooth muscle muscarinic cholinergic receptors or 5-HT receptors; contractions induced by electrical field stimulation (EFS) of enteric nerve terminals. Pair of platinum electrodes positioned parallel to the muscle strips and a Grass S88 stimulator (Grass Institute Division, W. Warwick, RI) were used to deliver 10-s trains of rectangular pulses with $0.5 \mathrm{~ms}$ pulse-duration applied at increasing frequencies of $1,5,10$ and $15 \mathrm{~Hz}$. Tetrodotoxin $(1 \mu \mathrm{M})$ which inhibits synaptic neurotransmission was added to the bathing solution to verify that the responses to EFS are neurally mediated. At the end of the experiment the muscle strips were blotted dry and weighed.

Solutions and drugs. The Krebs buffer was of the following composition $(\mathrm{mM}): \mathrm{NaCl} 120, \mathrm{KCl} 6, \mathrm{MgCl}_{2} 1.2$, $\mathrm{NaH}_{2} \mathrm{PO}_{4} 1.2, \mathrm{CaCl}_{2} 2.5, \mathrm{NaHCO}_{3} 14.4$ and glucose 11.5 (pH 7.3-7.4 when aerated with $95 \% \mathrm{O}_{2}$ and $5 \% \mathrm{CO}_{2}$ ). Tegaserod maleate was supplied by Novartis Pharmaceuticals (East Hanover, NJ). Omeprazole, indomethacin, carbamylcholine chloride, atropine sulfate, 5-hydroxytryptamine creatinine sulfate and tetrodotoxin were obtained from Sigma (St. Louis, MO). Drugs were added to the baths in volumes less that $1 \%$ of the total bath volume. Tegaserod and omeprazole were dissolved in propylene glycol and the rest of the drugs were dissolved in distilled water.

Data analysis and statistics. Muscle contractile responses were measured as changes in resting tension $(\mathrm{mN})$ and were normalized for $\mathrm{g}$ wet weight of tissue. Data are presented as means \pm SEM from preparations isolated from 5-6 animals in each experimental group. To establish the model indomethacin-induced abnormalities were evaluated by a comparison to naïve rats using Student t- test. The effects of omeprazole, tegaserod, the combined treatment and the effect of the vehicle were compared between the groups of indomethacin-treated rats using ordinary one-way ANOVA or non-parametric Kruskal-Willis ANOVA followed by Bonferroni's or the non-parametric Dunn's test for multiple comparison. Differences were considered significant at $\mathrm{P}<0.05$.

\section{RESULTS}

Protective effects against indomethacin-induced gastric mucosal injury. Gastric ulceration was detected along the surface of mucosal folds in the glandular part of the stomach but was absent in the fundus $6 \mathrm{~h}$ after administration 
of indomethacin. Mean values for the gastric mucosal ulceration index are presented in Fig. (1A). Mucosal MPO activity, measured as a marker of neutrophil infiltration, was significantly elevated in the glandular stomach of indomethacin treated rats $(201 \pm 28 \mathrm{ng} / \mathrm{g}$ tissue, $\mathrm{n}=6)$ compared to naïve rats $(23 \pm 3 \mathrm{ng} / \mathrm{g}$ tissue, $\mathrm{n}=6)$. Pretreatment of the rats with tegaserod, omeprazole or with a combination of both drugs significantly reduced the formation of gastric lesions compared to rats pretreated with vehicle only (Fig. 1A). However, despite the significant reduction in the ulceration index, some lesions were still present. Pretreatment with omeprazole and tegaserod significantly attenuated MPO activity to levels that were similar to naïve animals (Fig. 1B). No significant differences in MPO activity were found between the groups pretreated with omeprazole or tegaserod administered alone or in combination.
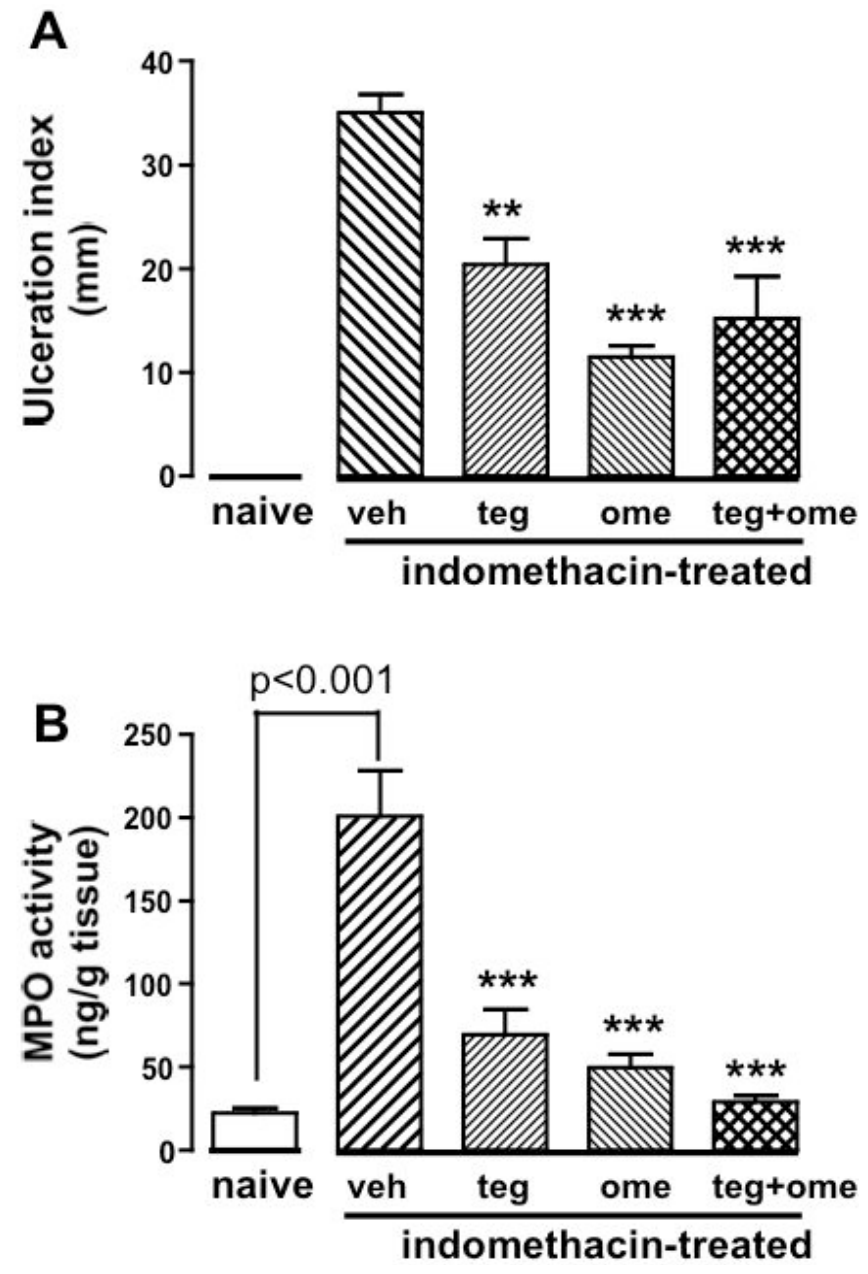

Fig. (1). Effect of pretreatment with tegaserod (1 mg/kg), omeprazole $(20 \mathrm{mg} / \mathrm{kg})$ or a combination of both drugs in a rat model of indomethacin-induced gastric injury. Mucosal ulceration (A) and myeloperoxidase activity. (B) Drugs were administered b.i.d. one day before the experiment and 30-min prior to indomethacin on the day of experiment. Gastric mucosal injury was evaluated $6 \mathrm{~h}$ after intragastric instillation of $50 \mathrm{mg} / \mathrm{kg}$ indomethacin. Data are mean \pm S.E.M. from 6 rats in each group. Statistical significance of differences between groups was assessed by one-way ANOVA followed by Bonferroni multiple comparison test. ** $\mathrm{P}<0.01$, *** $\mathrm{P}<0.001$ compared to the vehicle-treated group.
Protective effects against indomethacin-induced gastric muscle dysfunction. Circular smooth muscle strips isolated from the gastric antrum showed spontaneous contractile activity characterized by low basal tone $(200-500 \mathrm{mN} / \mathrm{g}$ tissue) and phasic contractions developing at a frequency of 4-6 per minute. The pattern of spontaneous activity was similar in all treatment groups.

Responses to high $\mathbf{K C l}$. Exposure to high $\mathrm{KCl}$ induced a tonic contraction in all circular muscle strips isolated from the gastric antrum. However, the maximal amplitude of $\mathrm{KCl}$ induced contractions was significantly higher in the strips isolated from indomethacin-treated rats undergoing pretreatment with the vehicle compared to naïve rats $(5810 \pm 610$ $\mathrm{mN} / \mathrm{g}, \mathrm{n}=6$ vs $3120 \pm 160 \mathrm{mN} / \mathrm{g}, \mathrm{n}=6, \mathrm{P}<0.05)$. Tegaserod and omeprazole administered individually showed a tendency to prevent the development of hypercontractile responses to $\mathrm{KCl}$ compared to the vehicle. When tegaserod and omeprazole were administered in combination, the $\mathrm{KCl}$-induced contractions showed significantly lower amplitude compared to the responses observed in vehicle-treated animals (Fig. 2).

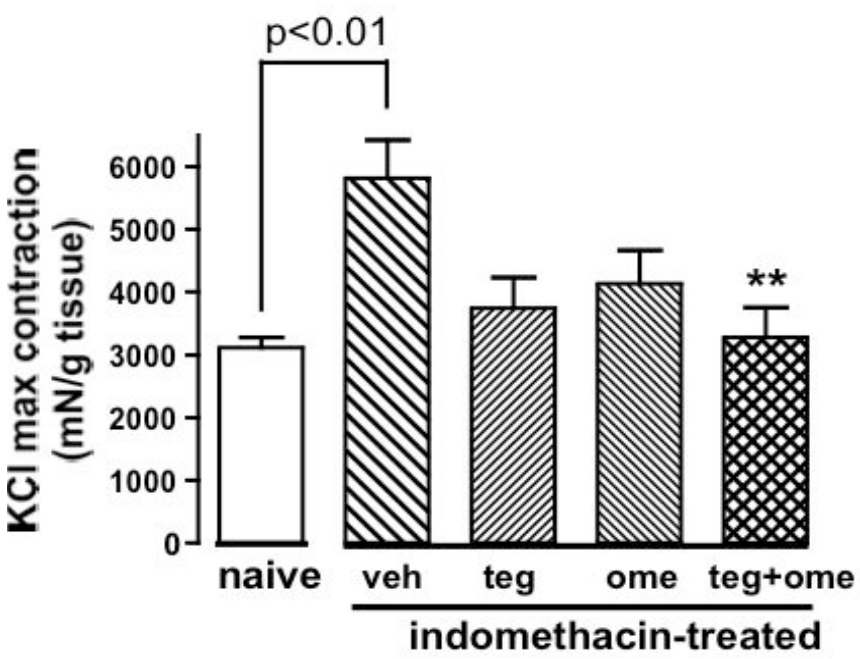

Fig. (2). Effect of pretreatment of the rats with tegaserod $(1 \mathrm{mg} / \mathrm{kg})$, omeprazole $(20 \mathrm{mg} / \mathrm{kg})$ or a combination of both drugs on the contractile response to $\mathrm{KCl}(80 \mathrm{mM})$ in circular muscle strips from the gastric antrum of rats with indomethacin-induced gastric damage. Data are mean \pm S.E.M. from 6 rats in each group. Statistical significance of differences between groups was assessed by one-way ANOVA followed by Bonferroni multiple comparison test. ** $\mathrm{P}<0.01$ compared to the vehicle-treated group.

Responses to carbachol. Circular muscle strips isolated from the gastric antrum of both indomethacin-treated and naïve rats responded to increasing concentrations of carbachol (1 nM-10 mM) added to the bathing solution with concentration-dependent contractions. Comparison of the carbachol concentration-response curves showed a significant increase in the amplitude of the contractile responses in muscle strips from indomethacin-treated rats which received vehicle compared to untreated naïve rats (Fig. 3). The increased muscle reactivity appeared without a significant shift in sensitivity to the muscarinic receptor agonist since similar $\mathrm{EC}_{50}$ values were obtained in naïve and indomethacin-treated rats (naïve rats: $\log \mathrm{EC}_{50}=-6.95,95 \% \mathrm{CL}-7.38$ to -6.51 , Hill slope 1.07 vs vehicle pretreated rats receiving indomethacin: $\log \mathrm{EC}_{50}=-7.32,95 \% \mathrm{CL}-7.62$ to -7.01 , Hill slope 1.11 ). Tegaserod or omeprazole administered individually or in 
combination did not show a significant shift in the concentration-effect curves to carbachol (tegaserod-treated rats: log $\mathrm{EC}_{50}=-6.93,95 \% \mathrm{CL}-7.17$ to -6.70 ; omeprazole-treated rats: $\log \mathrm{EC}_{50}=-7.60,95 \% \mathrm{CL}-7.82$ to -7.44 ; omeprazole plus tegaserod-treated rats: $\log \mathrm{EC}_{50}=-7.60,95 \% \mathrm{CL}-7.91$ to -7.22 ) (Fig. 3A). In contrast, pretreatment of the rats with the combination of tegaserod plus omeprazole resulted in a significant attenuation of maximal carbachol-induced muscle contraction compared to the effect of the vehicle (Fig. 3B).
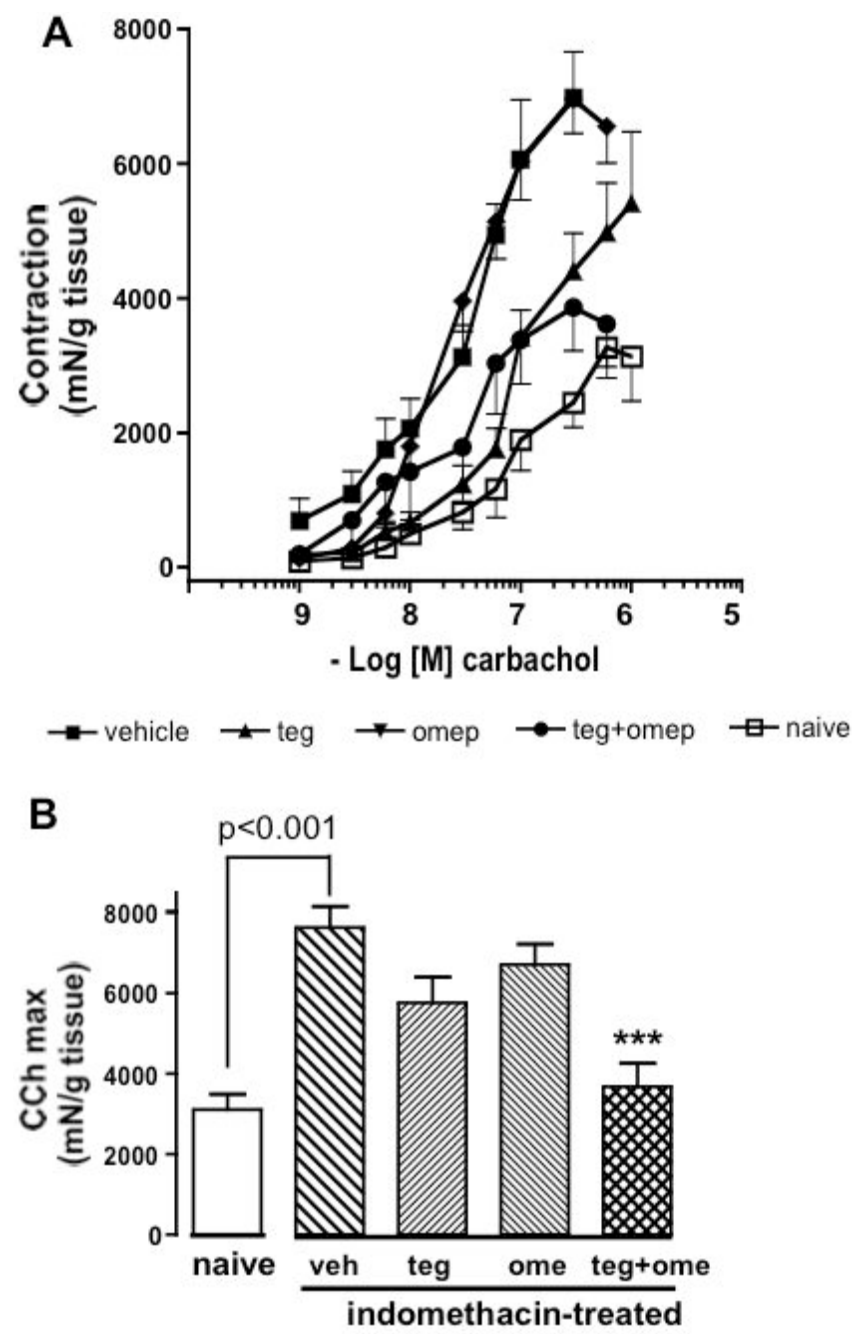

Fig. (3). Effect of pretreatment of the rats with tegaserod $(1 \mathrm{mg} / \mathrm{kg})$, omeprazole $(20 \mathrm{mg} / \mathrm{kg})$ or a combination of both drugs on the contractile responses to carbachol in circular muscle strips from the gastric antrum of rats with indomethacin-induced gastric damage. (A) Concentration-response curves obtained by cumulative addition of carbachol $(1 \mathrm{nM}-1 \mu \mathrm{M})$ to the bathing solution. (B) Maximal responses to carbachol. Data are mean \pm S.E.M. from 6 rats in each group. Statistical significance of differences between groups was assessed by Kruskal-Willis non-parametric ANOVA followed by Dunn's multiple comparison test. ${ }^{* * *} \mathrm{P}<0.001$ compared to vehicle-treated group.

Responses to 5-HT. Antral smooth muscle strips from naïve or indomethacin-treated rats responded to addition of 5-HT to the bathing solution with concentration-dependent contractions. The amplitude of contractile responses in muscle strips from naïve rats was significantly $(\mathrm{P}<0.05)$ lower compared to the responses in strips from indomethacin- treated rats receiving vehicle. However, there was no significant shift in the concentration response curve to 5-HT (log $\mathrm{EC}_{50}=6.23,95 \% \mathrm{CL}-7.10$ to -5.78 , Hill slope 0.48 in naïve rats vs $\log \mathrm{EC}_{50}=6.84,95 \% \mathrm{CL}-8.03$ to -5.81 , Hill slope 0.65 ) (Fig. 4A). The $\log \mathrm{EC}_{50}$ values for 5 -HT in rats treated with tegaserod, omeprazole or the combination were -7.11 (95\% CL -8.04 to -6.20$),-6.85$ (95\% CL -7.94 to -5.72$)$ and 6.51 (95\% CL -7.11 to -5.91$)$, respectively. Pretreatment of the rats with tegaserod alone or a combination of tegaserod and omeprazole caused a significant reduction in the amplitude of the maximal response to 5-HT compared to the effect of the vehicle (Fig. 4B). In comparison, the contractile response in strips from omeprazole-pretreated rats did not differ significantly from the response in preparations from vehicle-treated animals.
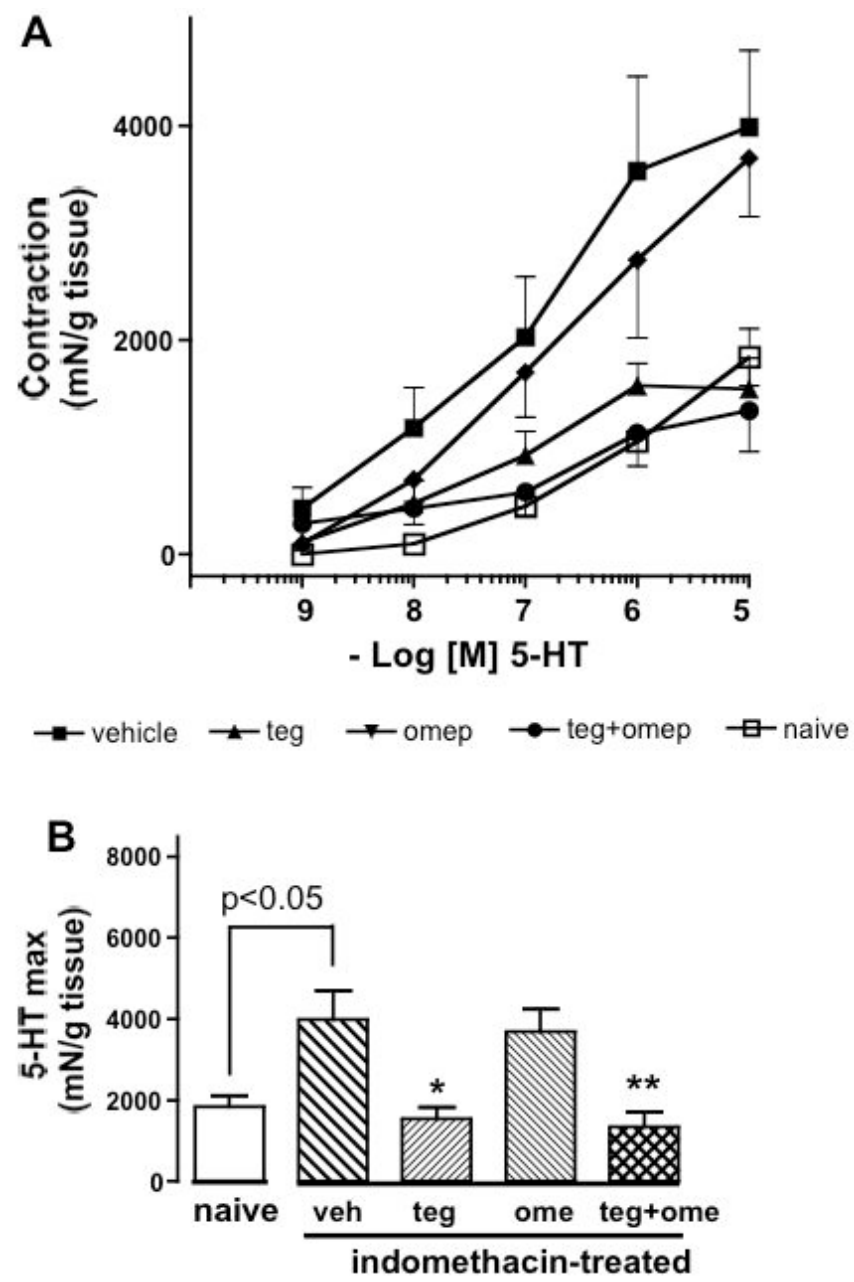

Fig. (4). Effect of pretreatment of the rats with tegaserod $(1 \mathrm{mg} / \mathrm{kg})$, omeprazole $(20 \mathrm{mg} / \mathrm{kg})$ or a combination of both drugs on the contractile responses to 5-HT in circular muscle strips from the gastric antrum of rats with indomethacin-induced gastric damage. (A) Concentration-response curves achieved by cumulative addition of 5-HT $(1 \mathrm{nM}-10 \mu \mathrm{M})$ to the bathing solution. (B) Maximal responses to 5-HT. Data are mean \pm S.E.M. from 6 rats in each group. Statistical significance of differences between groups was assessed by one-way ANOVA followed by Bonferroni multiple comparison test. * $\mathrm{P}<0.05,{ }^{*} * \mathrm{P}<0.01$ compared to vehicle-treated group.

Responses to electrical field stimulation (EFS). EFS applied at frequencies of $1-15 \mathrm{~Hz}$ induced frequencydependent responses characterized by an initial inhibition of 
spontaneous contractions or a low amplitude relaxation followed by a pronounced contraction. The inhibition persisted during stimulation while the contractile response developed after the end of the stimulus train. Since antral muscles isolated from all treatment groups and from naïve rats maintained low basal tone $(200-500 \mathrm{mN} / \mathrm{g}$ tissue) EFS-induced relaxations were negligible at all frequencies of EFS. The contractions were abolished by addition of tetrodotoxin (1 $\mu \mathrm{M})$ to the bathing solution, indicating that the EFS-induced responses are neurogenic. In muscle strips isolated from naïve rats EFS-induced the contractile responses increased linearly with the increase of stimulation frequency. A comparison between the amplitude of EFS-induced contractions in muscle strips from naïve and indomethacin-treated animals demonstrated a significant increase in the indomethacin-treated rats dosed with vehicle (Fig. 5).

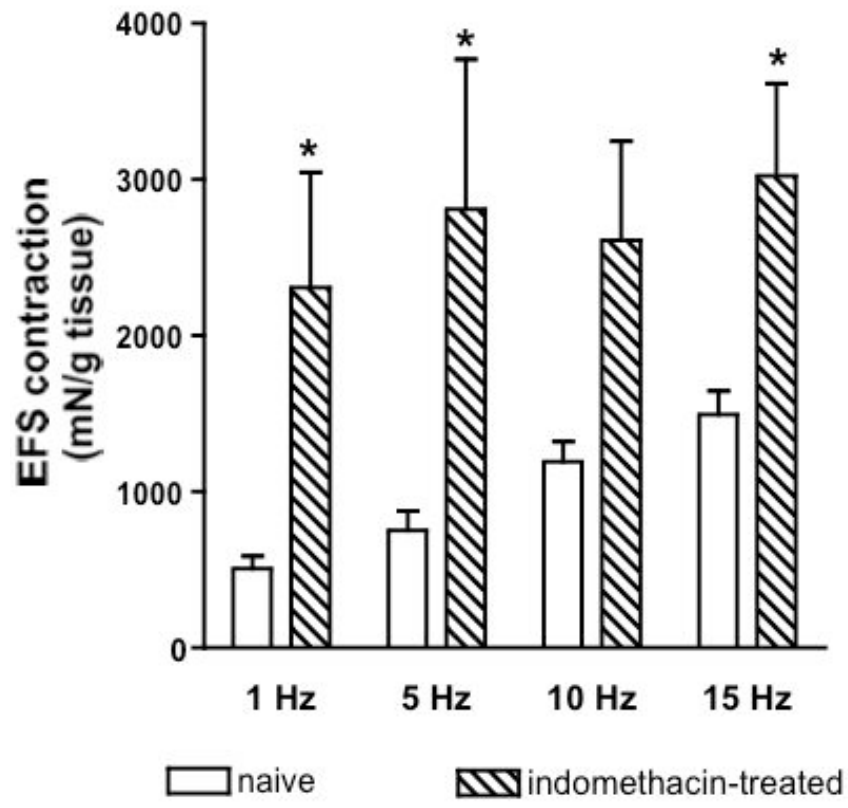

Fig. (5). Neurally mediated responses induced by EFS $(0.5 \mathrm{~ms}, 1-$ $15 \mathrm{~Hz}$ ) in circular muscle strips isolated from the gastric antrum of naïve rats or rats with indomethacin-induced gastric damage. Data are mean \pm S.E.M. from 6 rats in each group. Differences were assessed by one-way ANOVA followed by Bonferroni multiple comparison test. ${ }^{*} \mathrm{P}<0.05$ compared to responses in naïve rats induced by the same stimulus frequency.

Moreover, these preparations responded with contractions of relatively high amplitude to the lower stimulus frequency and showed no linear increase in amplitude with the increase in EFS frequency (Table 1, slope not significantly different from zero).

The increased amplitude of contractile responses to low stimulus frequency and the loss of the linear increase of contractions as a function of the frequency of stimulation suggest that the muscles from indomethacin-treated rats have developed hypercontractility and hypersensitivity to nerve stimulation. The effects of tegaserod and omeprazole on indomethacin-induced abnormalities in the responses to EFS were evaluated comparing the results obtained by linear regression of frequency-response data (Fig. 6). Pretreatment of the rats with tegaserod or the combination of tegaserod and omeprazole prevented the increase of contractile responses to EFS and preserved the linear frequency-response relation- ship (Table 1). Omeprazole applied individually preserved the linear frequency-response relationship, but was not able to prevent the increase in amplitude of the contractile responses (Fig. 6).

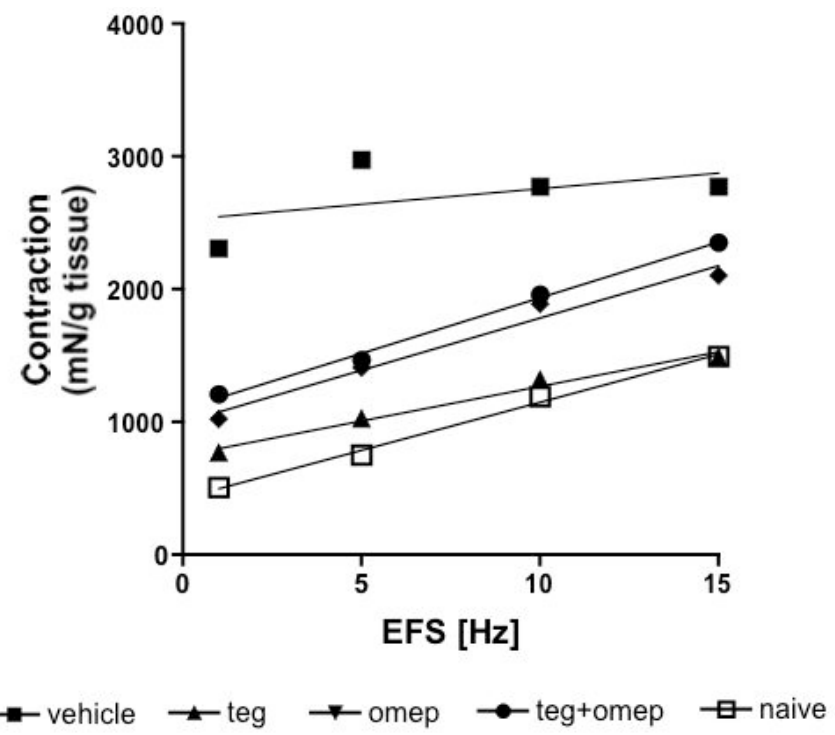

Fig. (6). Regression lines illustrating the frequency-dependent contractile responses to EFS $(0.5 \mathrm{~ms} .1-15 \mathrm{~Hz})$ in circular muscle strips from the gastric antrum. Pretreatment of the rats with tegaserod (1 $\mathrm{mg} / \mathrm{kg})$, omeprazole $(20 \mathrm{mg} / \mathrm{kg})$ or a combination of both drugs protected the linear increase in the amplitude of contractile responses with the increase in EFS frequency (slopes are presented in Table 1). Regression analysis performed on data from 6 rats in each group.

Table 1. Effect of Tegaserod and Omeprazole on the Linear Frequency-Response Relationship of the Contractile Responses Induced by EFS (0.5 ms, 1-15 Hz) in Antral Muscle Strips

\begin{tabular}{|c|c|c|c|c|}
\hline \multirow{2}{*}{ Treatment } & \multicolumn{5}{|c|}{ Regression Analysis } \\
\cline { 2 - 5 } & Slope & $\mathbf{r}^{\mathbf{2}}$ & $\mathbf{F}$ & $\mathbf{P}$ (Deviation from 0) \\
\hline \hline Nä̈ve rats & $72.3 \pm 5.1$ & 0.99 & 345.5 & 0.003 \\
\hline Rats with indomethacin-induced gastric injury \\
\hline vehicle & $25.5 \pm 2.8$ & 0.25 & 0.7 & 0.49 n.s \\
\hline tegaserod & $51.9 \pm 5.1$ & 0.98 & 102.6 & 0.009 \\
\hline omeprazole & $78.8 \pm 9.5$ & 0.97 & 67.8 & 0.01 \\
\hline $\begin{array}{c}\text { tegaserod and } \\
\text { omeprazole }\end{array}$ & $83.7 \pm 4.3$ & 0.99 & 379.9 & 0.002 \\
\hline
\end{tabular}

Regression analysis of data from 5-6 experiments per group; slopes are mean \pm SEM.

\section{DISCUSSION}

The results of the present study demonstrate that tegaserod, a prokinetic activating $5-\mathrm{HT}_{4}$ receptors, had a protective effect against indomethacin-induced gastric inflammation (increased MPO activity) and mucosal ulceration, which was comparable to the gastroprotective effect of the proton pump inhibitor omeprazole. In our experiments, indomethacin caused mucosal ulceration and a significant increase of neutrophil infiltration in the gastric antrum, but not in the fundus. Others have also noted [25] that NSAID-induced ulcera- 
tion is confined to the glandular stomach (gastric corpus, antrum and pyloric region) and that no visible lesions or significant increase in inflammatory cell infiltrates are present in the fundus. As a functional correlate of the mucosal lesions we observed an increased contractility in muscle strips from the gastric antrum. The muscles isolated from indomethacin-treated rats showed hypercontractility in response to high $\mathrm{K}^{+}$depolarization, carbachol, 5-HT or EFS of enteric nerves. Earlier studies implied that increased contractility of the antral muscle contributes significantly to development of NSAIDs-induced gastric mucosal damage, moreover, antral hypermotility itself was found to be sufficient to induce ulcerative damage to the gastric mucosa [26-28]. The mechanism responsible for the gastroprotective effect of tegaserod is unclear but it is most likely that it involves an effect on 5$\mathrm{HT}_{4}$ receptors regulating gastric contractility. Although, there is evidence that tegaserod may also act as inhibitor of $5-\mathrm{HT}_{1 \mathrm{~B}}[24]$ and $5-\mathrm{HT}_{2 \mathrm{~B}}$ receptors $[23,29]$, it is logical to assume that the activation of $5-\mathrm{HT}_{4}$ receptors which affects motility plays a role in the protective effect of tegaserod. This line of evidence is supported by the finding that stimulation of $5-\mathrm{HT}_{4}$ receptors by tegaserod inhibits contractility in human colonic circular muscle [30]. A recent study had shown that tegaserod treatment normalizes cholineregic hypercontractility of isolated duodenal muscle induced by high cholesterol diet in the ground squirrel [31].

Another important functional effect of tegaserod which could be relevant to our findings is its ability to enhance gastric emptying $[32,33]$. However, in our experiments it is unlikely that the gastroprotective effect of tegaserod results simply from an accelerated evacuation of indomethacin from the gastric lumen, since indomethacin is rapidly absorbed and high plasma levels are quickly reached after a single oral dose [34]. In the clinic the gastroprokinetic effect may prove beneficial in patients who are already using proton pump inhibitors, since both animal and human studies have shown that tegaserod can reverse the delay in gastric emptying induced by omeprazole $[19,35]$.

Mucosal blood flow is a major factor maintaining integrity of the gastric mucosal barrier to acid back diffusion and indomethacin treatment has been shown to have an adverse effect manifested by a reduction in gastric mucosal blood volume [36]. A potential additional mechanism that could play a role in the observed gatroprotective action of tegaserod in this rat model is an increase in gastric mucosal blood flow. Preliminary studies have demonstrated antagonist activity of tegaserod at $5-\mathrm{HT}_{1 \mathrm{~B}}$ receptors which can cause vasoconstriction [24].

In summary, our results demonstrate that the pharmacological targeting of peripheral $5-\mathrm{HT}_{4}$ serotonin receptors has a protective effect against gastric muscle dysfunction and muscosal damage induced by indomethacin in the rat. The effect of tegaserod is most likely due to activation of $5-\mathrm{HT}_{4}$ receptors in the stomach which prevent the development of indomethacin-induced smooth muscle hypercontractility. Our findings suggest that prokinetics acting via serotoninergic mechanisms may have a protective effect against NSAID-induced dysfunction and mucosal injury. In this study, the gastroprotective effect of tegaserod was comparable with the effect of omeprazole, a proton pump inhibitor used clinically as concurrent gastroprotective therapy in high-risk patients receiving NSAIDs. Despite the fact that the marketing of tegaserod has been suspended, due to cardiovascular ischemic events developing as side effects, the clinical implications of the prokinetic action of serotoninergic drugs which activate $5-\mathrm{HT}_{4}$ mechanisms in the stomach deserves further investigation [21]. Since both NSAIDs and omeprazole reduce the rate of gastric emptying, it is possible that a $5-\mathrm{HT}_{4}$ agonist with prokinetic activity may have an added beneficial effect in patients using omeprazole to protect against gastric mucosal injury when high doses or multiple NSAIDs are required.

\section{ACKNOWLEDGEMENT}

Financial support for this study was provided by Novartis Pharmaceutical Corp.

\section{REFERENCES}

[1] Whittle, B.J. Prostaglandins, 1981, 21, 113.

[2] Kargman, S.; Charleson, S.; Cartwright, M.; Frank, J.; Riendeau, D.; Mancini, J.; Evans, J.; O'Neill, G. Gastroenterology, 1996, 111, 445 .

[3] Wallace, J.L.; McKnight, W.; Reuter, B.K.; Vergnolle, N. Gastroenterology, 2000, 119, 706.

[4] Tanaka, A.; Araki, H.; Komoike, Y.; Hase, S.; Takeuchi, K. J. Physiol. Paris, 2001, 95, 21.

[5] Takeuchi, K.; Ueshima, K.; Hironaka, Y.; Fujioka, Y.; Matsumoto, J.; Okabe, S. Digestion, 1991, 49, 175.

[6] Santucci, L.; Fiorucci, S.; Di Matteo, F.M.; Morelli, A. Gastroenterology, 1995, 108, 393

[7] Souza, M.H.; Troncon, L.E.; Cunha, F.Q.; Oliveira, R.B. Braz. J. Med. Biol. Res., 2003, 36, 1383.

[8] Santos, C.L.; Medeiros, B.A.; Palheta-Junior, R.C.; Macedo, G.M.; Nobre-e-Souza, M.A.; Troncon, L.E.; Santos, A.A.; Souza, M.H. Neurogastroenterol. Motil., 2007, 19, 225.

[9] Takeuchi, K.; Okada, M.; Niida, H.; Okabe, S. Dig. Dis. Sci., 1990, 35,984 .

[10] Takeuchi, K.; Tanaka, A.; Hayashi, Y.; Kubo, Y. Dig. Dis. Sci., 2004, 49, 180.

[11] Narita, T.; Okabe, N.; Hane, M.; Yamamoto, Y.; Tani, K.; Naito, Y.; Hara, S. J. Vet. Pharmacol. Ther., 2006, 29, 569.

[12] Hawkey, C.J.; Karrasch, J.A.; Szczepanski, L.; Walker, D.G.; Barkun, A.; Swannell, A.J.; Yeomans, N.D. N. Engl. J. Med., 1998, 338, 727.

[13] Chan, F.K.; Ching, J.Y.; Hung, L.C.; Wong, V.W.; Leung, V.K.; Kung, N.N.; Hui, A.J.; Wu, J.C.; Leung, W.K.; Lee, V.W.; Lee, K.K.; Lee, Y.T.; Lau, J.Y.; To, K.F.; Chan, H.L.; Chung, S.C.; Sung, J.J. N. Engl. J. Med., 2005, 352, 238.

[14] Takeuchi, K.; Tanaka, A.; Hayashi, Y.; Kubo, Y. Dig. Dis. Sci. 2004, 49, 180.

[15] Rasmussen, L.; Oster-Jorgensen, E.; Qvist, N.; Kraglund, K.; Hovendal, C.; Pedersen, S.A. Aliment. Pharmacol. Ther., 1991, 5, 85.

[16] Rasmussen, L.; Oster-Jorgensen, E.; Qvist, N.; Pedersen, S.A. Scand. J. Gastroenterol., 1999, 34, 671.

[17] Benini, L.; Castellani, G.; Bardelli, E.; Sembenini, C.; Brentegani, M.T.; Caliari, S.; Vantini, I. Dig. Dis. Sci., 1996, 41, 469.

[18] Parkman, H.P.; Urbain, J.L.; Knight, L.C.; Brown, K.L.; Trate, D.M.; Miller, M.A.; Maurer, A.H.; Fisher, R.S. Gut, 1998, 42, 243.

[19] Tougas, G.; Earnest, D.L.; Chen, Y.; Vanderkoy, C.; Rojavin, M. Aliment. Pharmacol. Ther., 2005, 22, 59.

[20] Galligan, J.J.; Vanner, S. Neurogastroenterol. Motil., 2005, 17, 643

[21] Pasricha, P.J. Gastroenterology, 2007, 132, 2287.

[22] Camilleri, M. Aliment. Pharmacol. Ther., 2001, 15, 277.

[23] Beattie, D.T.; Smith, J.A.; Marquess, D.; Vickery, R.G.; Armstrong, S.R.; Pulido-Rios, T.; McCullough, J.L.; Sandlund, C.; Richardson, C.; Mai, N.; Humphrey, P.P. Br. J. Pharmacol., 2004, 143, 549.

[24] Weber, E.; Hamel, C.T.; Bernhard, M.; Phannkuche, H.J. Gastroenterology, 2006, 130, A489.

[25] Morini, G.; Grandi, D.; Arcari, M.L.; Bertaccini, G. Aliment. Pharmacol. Ther., 1995, 9, 615.

[26] Takeuchi, K.; Ueki, S.; Okabe, S. Dig. Dis. Sci., 1986, 31, 1114. 
[27] Okada, M.; Niida, H.; Takeuchi, K.; Okabe, S. Dig. Dis. Sci., 1989, 34, 694.

[28] Mersereau, W.A.; Hinchey, E.J. Am. J. Physiol., 1982, 242, G429.

[29] McCullough, J.L.; Armstrong, S.R.; Hegde, S.S.; Beattie, D.T. Pharmacol. Res., 2006, 53, 353.

[30] Tam, F.S.; Hillier, K.; Bunce, K.T. Br. J. Pharmacol., 1994, 113, 143.

[31] Mathison, R.; Shaffer, E. B.M.C. Gastroenterol., 2006, 6, 8.

[32] Degen, L.; Matzinger, D.; Merz, M.; Appel-Dingemanse, S.; Osborne, S.; Luchinger, S.; Bertold, R.; Maecke, H.; Beglinger, C. Aliment. Pharmacol. Ther., 2001, 15, 1745.
[33] Degen, L.; Petrig, C.; Studer, D.; Schroller, S.; Beglinger, C. Neurogastroenterol. Motil., 2000, 17, 821 .

[34] Ogiso, T.; Iwaki, M.; Tamaki, E. J. Pharmacobiodyn., 1983, 6 803.

[35] Cowan, A.; Earnest, D.; Ligozio, G.; Rojavin, M.A. Eur. J. Pharmacol., 2005, 517, 127.

[36] Yokoyama, T.; Kitazawa, T.; Ishii, A.; Ogihara, T.; Sato, N.; Karasawa, A. Jpn. J. Pharmacol., 1994, 65, 171. 